\title{
Au-delà du mur : Israël face à sa frontière
}

\section{Pierre Renno}

\section{(2) OpenEdition \\ Journals}

\section{Édition électronique}

URL : http://journals.openedition.org/echogeo/2289

DOI : 10.4000/echogeo.2289

ISSN : 1963-1197

\section{Éditeur}

Pôle de recherche pour l'organisation et la diffusion de l'information géographique (CNRS UMR 8586)

\section{Référence électronique}

Pierre Renno, «Au-delà du mur : Israël face à sa frontière », EchoGéo [En ligne], Sur le Vif, mis en ligne le 11 mars 2008, consulté le 19 avril 2019. URL : http://journals.openedition.org/echogeo/2289 ; DOI : 10.4000/echogeo.2289

Ce document a été généré automatiquement le 19 avril 2019

Licence Creative Commons

EchoGéo est mis à disposition selon les termes de la licence Creative Commons Attribution - Pas d'Utilisation Commerciale - Pas de Modification 4.0 International 


\title{
Au-delà du mur : Israël face à sa frontière
}

\author{
Pierre Renno
}

1 Là où le français ne dispose que du substantif «frontière », la langue anglaise distingue entre la frontière - frontier et la frontière - border. Depuis le traité de Westphalie et l'affirmation de l'Etat souverain comme monade du système international, les frontières que connaît l'Europe sont des borders, des lignes séparant des entités étatiques souveraines.

2 A l'inverse de la frontière - border, la frontière - frontier n'est pas une ligne, mais une région. La notion de frontier a été utilisée pour décrire certaines zones en cours de colonisation par des populations s'étendant progressivement sur des territoires. On trouvait ainsi une frontier au XIX ${ }^{\mathrm{e}}$ dans les vastes étendus d'Australie, de Sibérie, du Brésil et bien sûr des Etats-Unis. La frontier désignait alors un entre-deux, une région intermédiaire entre l'espace apprivoisé, colonisé, contrôlé et l'inconnu. Plus tout à fait sauvage, mais pas encore complètement domptée, la frontière (frontier) était cette zone grise, en passe d'être conquise et civilisée. La frontière est donc une zone de tension; elle est l'expression spatiale d'un combat dont la figure centrale est le pionnier. C'est en effet le pionnier, qui par sa présence, fonde la frontière.

3 Selon la définition retenue par Yiftachel et Fenster, la frontière - frontier est une zone dans laquelle des pionniers / colons (settlers) mènent une politique destinée à s'approprier les ressources, principalement territoriales, des indigènes ${ }^{1}$. Nous ajouterons que ce type de frontière - frontier se construit sur un rapport de force asymétrique. Historiquement, son apparition est intimement liée à celle du mouvement colonial. Typiquement, la frontière - frontier est le dispositif territorial qui s'est mis en place lorsque des populations européennes émigrées sont entrées en contact avec des populations indigènes.

4 Espace en transition, la frontière (frontier) est par définition appelée à être intégrée, incorporée et assimilée. La zone pionnière peut alors être rejetée plus loin, vers une périphérie plus lointaine, jusqu'alors perçue comme sauvage et inaccessible. 


\section{frontiers}

$5 \mathrm{Si}$, dans la période pré-étatique, la plaine côtière s'affirmait comme le cœur économique et démographique du foyer national juif en devenir, le Néguev et la Galilée devinrent, eux, des frontiers, l'espace d'expression du pionniérisme sioniste (l'hityashvut). Dans les espaces urbains, où communautés juives et arabes vivaient côte à côte, l'économie de marché rendait difficile les perspectives de séparation ethnique. C'est seulement sur la frontier que les sionistes socialistes purent créer de micro-sociétés entièrement coupées des populations palestiniennes - y compris professionnellement ${ }^{2}$ - et promouvoir leur projet de renouvellement identitaire ${ }^{3}$.

6 Même si les déplacements de population consécutifs au conflit de 1948 - expulsion des Palestiniens et arrivée d'immigrants juifs - consolidèrent l'assise démographique et territoriale du jeune Etat d'Israël, le Néguev et la Galilée ont continué d'être perçus comme des frontières (frontiers) - à ce titre sujettes à des politiques d'implantation juive alors même que ces espaces faisaient partie intégrante de l'Etat et que les populations palestiniennes qui y résidaient s'étaient vues accorder la citoyenneté israélienne.

7 A la suite du conflit de 1967, l'occupation militaire du Sinaï, du Golan, de la Cisjordanie et de la bande de Gaza, a contribué à ouvrir de nouveaux espaces pionniers. Ceux-ci ont permis un renouvellement, tant géographique que culturel, du pionniérisme israélien : les pionniers du Gouch Emounim, partis à la conquête des monts de Judée-Samarie ${ }^{4}$, prétendaient en effet être porteurs, dans la lignée des sionistes socialistes du début du siècle, d'un nouvel ethos israélien centré, lui, sur le retour aux commandements divins.

8 S'il s'est exprimé pendant près de 30 ans dans la bande de Gaza, ce nouveau pionniérisme s'est surtout concentré sur la Cisjordanie. Ce territoire présentait en effet un certain de nombre de vacuums démographiques propices à l'implantation. Il était également, pour les colons néo-sionistes, parsemé de sites bibliques qui se prêtaient à un investissement symbolique.

9 D'abord hésitant, le gouvernement israélien a, après l'alternance de 1977, activement soutenu cette politique d'implantation. Le projet sioniste se relançait ainsi dans la conquête d'un territoire hostile - il ouvrait une nouvelle frontière (frontier).

Deux Intifada et un processus de paix avorté plus tard, la frontier ouverte en 1967 est toujours là : les pionniers néo-sionistes s'approprient, dunam après dunam ${ }^{5}$, les collines de Cisjordanie. A coup de grands complexes résidentiels ou de petits points de peuplement d'une dizaine de caravanes, ils reproduisent ou renouvellent les pratiques de la frontier des pionniers du début du siècle.

11 C'est ce paysage de frontière - frontier qui a vu s'ériger, depuis $2002^{6}$, un édifice qui est lui typiquement associé à l'idée de frontière - border : le Mur de séparation ${ }^{7}$.

Avant de s'imposer à l'agenda politique, l'idée de clôture de sécurité fut tout d'abord un projet porté par certains groupes entrepreneurs d'opinion ${ }^{8}$. Ceux-ci ont pu s'appuyer sur le sentiment, assez répandu dans l'opinion publique israélienne, que ni les négociations de paix, ni les opérations militaires n'étaient en mesure de mettre un terme aux actions terroristes - particulièrement nombreuses dans les premières années de la seconde Intifada. Dès lors, la clôture apparaissait comme la seule solution permettant d'interdire aux terroristes l'accès aux grands centres urbains israéliens. Sa popularité était telle que certains politiques s'accaparèrent le projet $^{9}$. Dès lors, la pression populaire sur le gouvernement d'union nationale alors aux affaires balaya les différentes réticences. 
13 Malgré les recours devant le Cour International de Justice ${ }^{10}$ et la Cour Suprême israélienne ${ }^{11}$, la construction du mur de séparation s'est poursuivie lors des six dernières années. Même s'il est difficile d'obtenir des autorités israéliennes des détails quant à l'avancement des travaux, les nombreuses ONG qui documentent la situation s'accordent pour dire que seuls les deux tiers de cet édifice, dont la longueur prévu était de quelques $700 \mathrm{~km}$, ont aujourd'hui été achevés. Dans les zones rurales, le « mur » s'apparente en fait à une clôture équipée de détecteurs électroniques. Ce n'est qu'aux abord des espaces urbanisés de Tulkarem, Qalqilya, ou de Jérusalem, qu'il prend la forme d'un mur de béton de près de 8 mètres de haut.

Figure 1 - Autour de Bethlehem

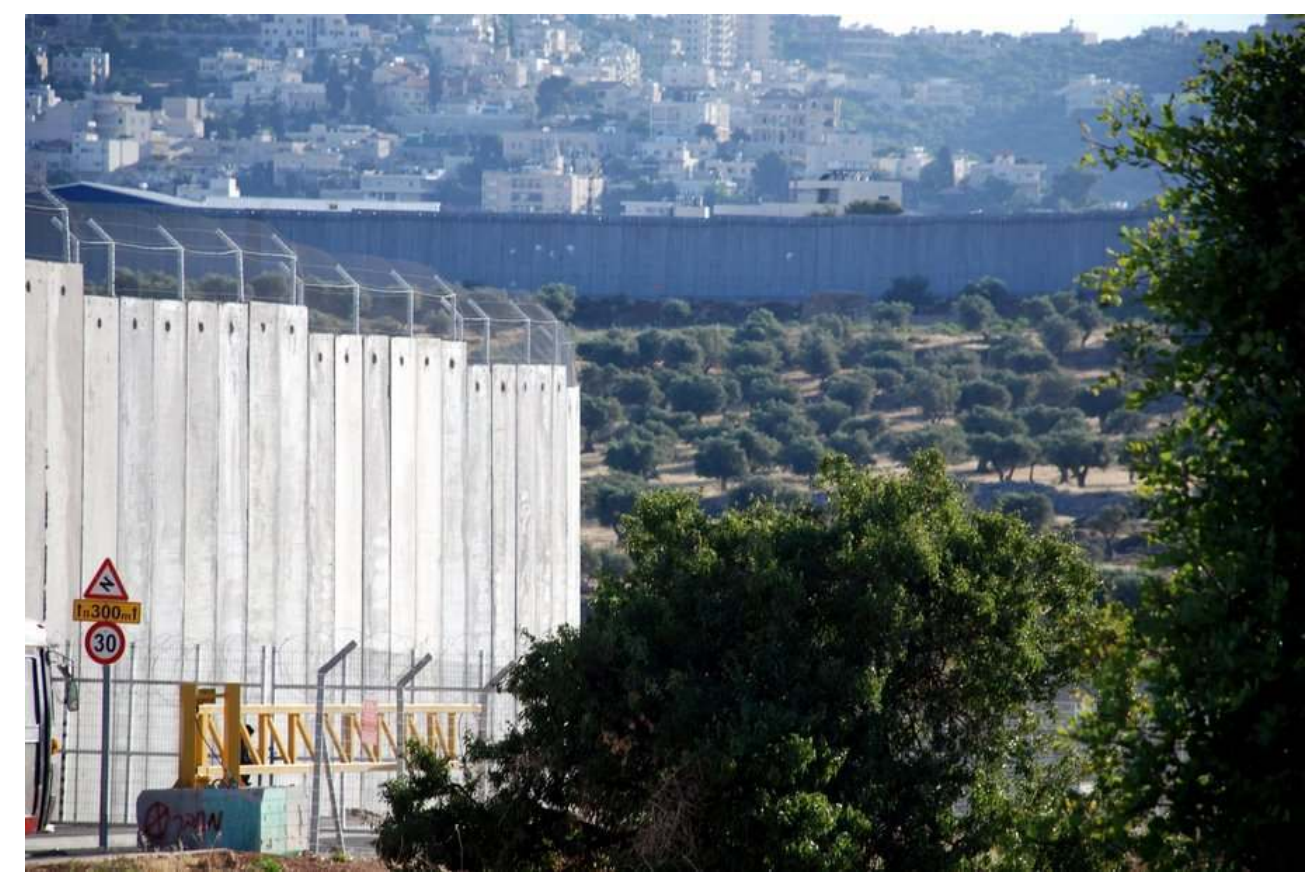

(Cliché de Pierre Renno,2007) 
Figure 2 - Dans une zone rurale du nord de la Cisjordanie

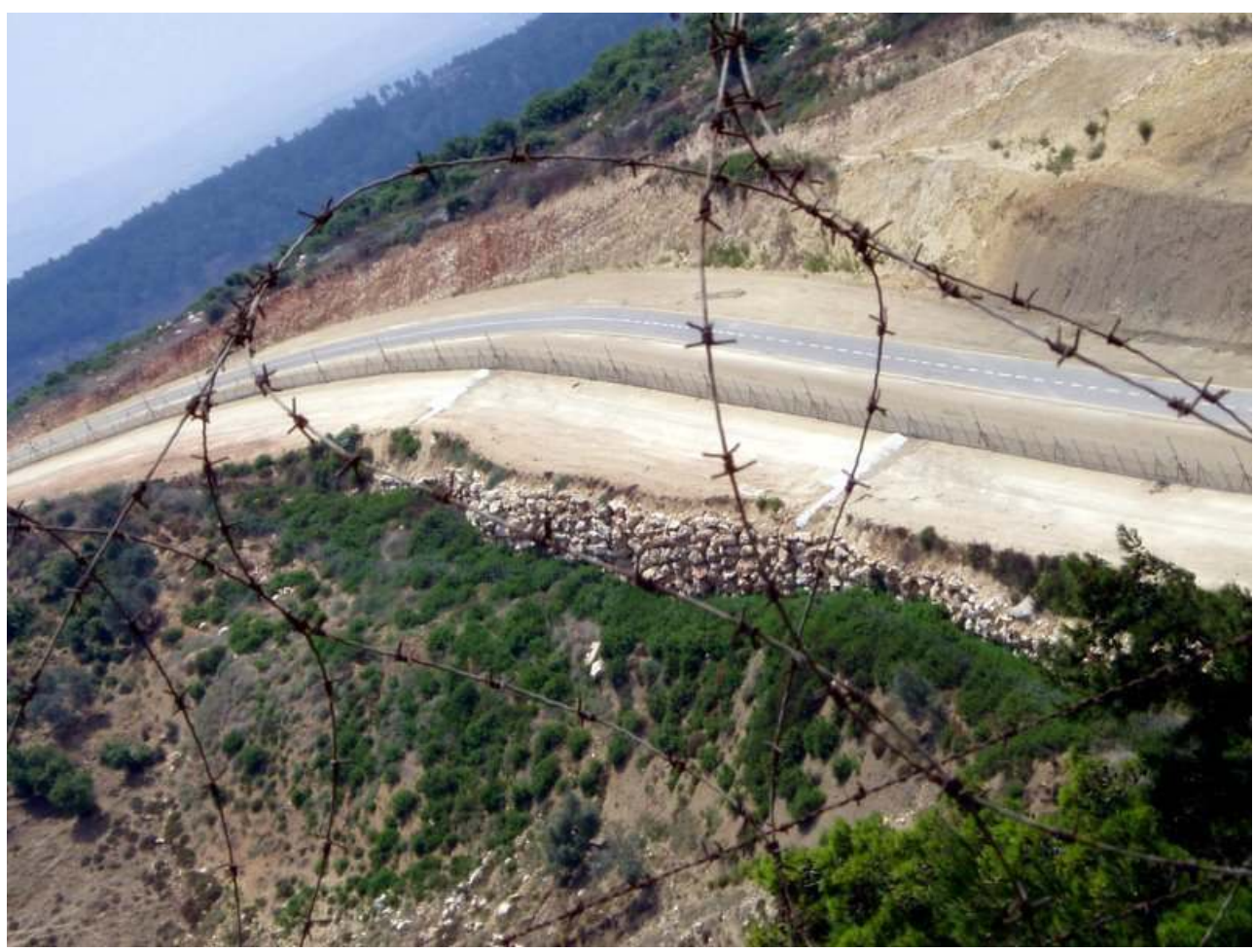

(Cliché de Pierre Renno,2006)

14 Cependant, la construction de ce mur de séparation n'a pas été accompagnée d'un retrait de l'armée et des colons - sur le modèle de celui effectué à Gaza à l'été 2005. Sur le terrain, le glissement de la frontier vers la border que laissait entrevoir la décision de 2002 ne s'est jamais concrétisé. Comme l'analyse Cédric Parizot, ce qu'on peut à l'heure actuelle observer en Cisjordanie est une juxtaposition de régimes territoriaux ${ }^{12}$. Le mur s'est imposé comme un instrument supplémentaire de la politique de contrôle des populations palestiniennes. Il n'a pas fait apparaître une séparation de type border, mais a au contraire contribué à renforcer une pénétration territoriale qui renvoie elle aux pratiques de la frontier.

15 Pour analyser la persistance de la frontière (frontier), il est aisé de s'en remettre à des considérations sécuritaires. On explique alors que le maintien de l'armée et des colons de l'autre côté du mur de séparation relèvent d'une stratégie de « défense à l'avant ", qu'elle permet à l'Etat d'Israël de «traiter la menace terroriste palestinienne au plus près ». De fait, alors même que, depuis quatre ans, l'intensité de l'Intifada a largement décru, les centres urbains palestiniens de Cisjordanie continuent d'être l'objet d'incursions régulières. Le précédent gazaouite rentrerait également dans cette équation : à Gaza, le retrait unilatéral effectué en 2005 s'est en effet révélé, d'un point de vue israélien, assez désastreux. Il n'a pas permis de mettre un terme aux attaques de rockets sur les villes environnantes et a même facilité la prise du pouvoir par le Hamas.

16 Cependant, derrière ces considérations sécuritaires immédiates se trouvent des questions de portée autrement plus significative. A long terme, les conséquences de la fermeture de la frontier ne seraient pas seulement sécuritaires, mais également identitaires.

Frontier 
17 Dans le modèle de l'Etat westphalien, doté de frontières fixes (borders), le peuple citoyen, le demos grec, tend à être défini territorialement comme l'ensemble des individus résidant dans les frontières de cet Etat. A l'inverse, la population d'un Etat ayant une frontière ( frontier) ouverte (les anglais parlent pour ce type d'Etat de settlement state - qu'on peut traduire par Etat pionnier) n'est pas prioritairement définie territorialement, mais ethniquement, c'est la population pionnière, en opposition à la population indigène.

L'existence d'une frontier ouverte contribue en fait à inverser le rapport entre population et territorialité. Une population ne devient pas citoyenne de par sa résidence dans le territoire de l'Etat, mais un territoire devient étatique de par sa colonisation par une population ethniquement identifiée au groupe pionnier. La logique juridique qui prévaut dans les territoires occupés est ainsi que l'Etat d'Israël s'étend là où se trouvent les Juifs. Ainsi, juridiquement, alors que la Cisjordanie est sous administration militaire (et n'est de ce fait pas considérée comme un territoire israélien), les implantations juives de cette zone sont elles soumises au droit civil israélien - elles ne sont, légalement parlant, pas différentiable des collectivités locales situées à l'intérieur des frontières de $19677^{13}$.

19 Si les territoires occupés constituent une expression particulièrement brute du fondement ethnique sur lequel se définit la citoyenneté au sein d'un Etat pionnier, l'esprit de la frontier ne se limite pas à cette seule frontière externe, mais s'immisce également au cœur même de l'Etat d'Israël, dans ce que certains auteurs appellent « Israel proper » (en référence à ce qu'était, à la veille du conflit de 1967, l'entité territoriale israélienne).

Ainsi, dans les frontières (frontiers) internes de l'Etat d'Israël (principalement la Galilée et le Néguev), les autorités israéliennes travaillent à l'inverse à mettre les Palestiniens pourtant légalement citoyens israéliens - en marge du corps social. Pour ce faire les politiques nationales israéliennes s'ingénient à faire des espaces peuplés par les Arabes israéliens des territoires d'exception, ce qu'on pourrait appeler des infra-espaces.

Dans son article sur la naissance d'une minorité piégée, Adriana Kemp montre ainsi comment la logique des textes juridiques plaçant les Palestiniens citoyens d'Israël sous administration militaire (entre 1948 et 1966) se voulait territoriale. Pour éviter d'être jugées discriminatoires, ces lois ne définissaient pas une population se devant d'être contrôlée pour son appartenance ethnique, mais des zones à l'intérieur desquelles, pour des raisons de sécurité, l'armée était habilitée à restreindre les libertés des habitants. Sans surprise, ces zones «incluaient un nombre aussi élevé que possible de localités palestiniennes et excluait un nombre aussi élevé que possible de localités juives $»{ }^{14}$

Si l'administration militaire a pris fin il y a plus de 40 ans, la logique consistant à construire un infra-espace arabe israélien pour mettre sa population à l'écart du peuple citoyen est toujours à l'œuvre. Cette logique peut notamment se lire, en creux, dans la manière dont les politiques de développement territorial israéliennes contournent les agglomérations arabes israéliennes. Ainsi, en 1998, la zone de développement prioritaire aleph (une zone à l'intérieur de laquelle les particuliers et entreprises peuvent obtenir d'importantes réductions d'impôt) était découpée de telle manière qu'elle ne concerne que 4 localités arabes, contre 425 localités juives ${ }^{15}$ - ce alors même qu'elle est officiellement destinée à soutenir le développement des régions périphériques, au premier rang desquelles la Galilée et le Néguev. De fait, ces aides concernent les seules villes et villages juifs de ces régions majoritairement peuplés d'Arabes israéliens. 
23 Là encore, la définition d'un territoire étatique de plein droit apparaît inféodée à la présence sur ce territoire d'une population appartenant au groupe pionnier - l'espace étatique peuplé par les indigènes apparaissant alors comme un infra-espace. On comprend alors qu'Israël présente, à l'intérieur même des frontières (border) de 1967, des tendances propres à un settlement state, à un Etat dont le corps politique n'est pas l'ensemble des individus résidant sur son territoire, mais les seuls individus appartenant au groupe pionnier/non-indigène.

Pour Israël, adopter des frontières fixes (de type border), c'est donc aussi prendre le risque de glisser d'un modèle identitaire vers l'autre, d'un Etat défini ethniquement comme appartenant au groupe pionnier vers un Etat appartenant à l'ensemble de ses citoyens définis comme le groupe des individus résidant à l'intérieur des frontières (borders) de l'Etat.

26 Faire du mur de séparation une frontière (border) implique donc non seulement de renoncer à la Cisjordanie, mais aussi, à terme, de faire de tous ceux qui se trouvent à l'ouest de cette frontière (border) des citoyens israéliens de plein droit. Or, la perspective de pérenniser la présence d'une minorité arabe représentant aujourd'hui quelque $20 \%$ des Israéliens, est, pour une majorité d'Israéliens, particulièrement anxiogène. D'où ces propositions récurrentes de rattacher les régions peuplées d'Arabes israéliens qui peuvent géographiquement l'être à un éventuel futur Etat Palestinien. Le transfert territorial du Petit Triangle à la Palestine est ainsi évoqué ${ }^{16}$. Cette proposition consiste en fait à pousser à son terme la logique de marginalisation territoriale du secteur arabe israélien en cherchant tout bonnement à l'exclure du territoire national.

Si ce transfert territorial reste très hypothétique - puisque pour l'heure rejeté et par les négociateurs palestiniens, et surtout, par les citoyens arabes israéliens concernés ${ }^{17}-$ l'administration israélienne construit en ce moment la situation kafkaïenne à venir des populations palestiniennes résidant entre la Ligne Verte et le Mur de séparation ${ }^{18}$. Coupés des villes de Cisjordanie, ces Palestiniens (estimés entre 60000 et 100000 individus) se voient pour l'instant refuser tout statut (résidence ou citoyenneté) en Israël. Leur cas laisse présager la création d'un nouvel infra-espace palestinien à l'intérieur des futures frontières (borders) israéliennes.

On retrouve ici et la crainte israélienne de voir les frontières fixes (border) à venir définir un demos israélien comprenant une substantielle minorité arabe israélienne et surtout la tentation d'évacuer ces populations par la mise en place de régimes territoriaux d'exception.

29 Alors qu'il était Premier Ministre, Ehud Barak avait affirmé que le processus de paix devait conduire à la création d'un Etat palestinien au côté d'un Etat juif, et non d'un Etat palestinien au côté d'un Etat binational. Cette revendication, à l'heure où près de $20 \%$ des citoyens israéliens sont palestiniens, laisse entrevoir l'équilibre précaire sur laquelle est bâtie la posture de négociation israélienne, entre le désir de pérenniser une frontière ( border) et celui de conserver une définition ethnique de la citoyenneté héritée de l'idéologie et des pratiques de la frontière (frontier). 


\section{NOTES}

1. O. Yiftachel and T. Fenster, Frontier Development and Indigenous Peoples, Pergamon, 1997

2. Sur ce point, la lutte (connue sous le nom de bataille pour le « travail hébreu ») fut particulièrement âpre, la logique économique justifiant, comme en ville, le recours à une main d'œuvre arabe.

3. Voir Gershon Shafir ; « Land, Labor and the Origins of the Israeli-Palestinian Conflict, 1882 - 1914 ; University of California press ; 1996

4. Appellation néo-sioniste de la Cisjordanie.

5. Le dunam est l'unité de mesure spatiale utilisée en Israël. 1 dunam équivaut à $1000 \mathrm{~m}^{2}$

6. C'est le 14 avril 2002 que le gouvernement a officiellement approuvé la construction de cet édifice.

7. Inévitablement, ce nouvel arrivant sur la scène proche-orientale a donné lieu à une controverse sémantique. Alors que les Palestiniens y voient "a wall" (un mur), "a segregation wall", voire "an apartheid wall", les Israéliens préfèrent le qualifier de "fence" (clôture, Gader en hébreu), de "security fence" (cloture de sécurité) ou de "separation fence". Dans le cadre de l'analyse ici menée, nous avons choisi de faire référence au "Mur de séparation ", qui est l'appellation la plus usitée en français.

8. Parmi les plus influents, on peut citer l'association "Clôture pour la vie" de l'avocat Ilan Tzion, l'association Hipardout (séparation), fondée en 2001 par le physicien Yehiam Prior, ou encore le "Comité pour la construction d'un mur de sécurité", présidé par un ancien conseiller national à la sécurité, le général Uzi Dayan.

9. Au sein du Parti travailliste, c'est Haïm Ramon qui fit office de Cheval de Troie, obligeant le ministre de la défense Benyamin Ben-Eliezer à modifier son discours.

10. Le 9 juillet 2004, la Cour International de Justice l'a jugé contraire au Droit International et a demandé son démantèlement.

11. Pour la Cour Suprême israélienne, le Mur de séparation est un ouvrage sans portée politique. La Cour s'est donc contentée de juger de la légitimité du tracé en fonction des exigences sécuritaires et du tort causé aux Palestiniens. Elle a, à l'heure actuelle, exigé des modifications de tracé sur près de $60 \mathrm{~km}$.

12. Cédric Parizot ; « Après le Mur, quelles séparations ? »; conférence réalisée au Centre de Recherche Français de Jérusalem le 13 février 2008.

13. C'est là une des observations sur lesquelles Oren Yiftachel bâtit son concept théorique d'ethnocratie.

Oren Yiftachel ; “Ethnocracy': The Politics of Judaizing Israel/Palestine" Constellations 6 (3) , p. 364-390; 1999

Pour décrire cette réalité, Amnon Rubinstein, ancien ministre et juriste de formation, a façonné le terme de justice d'enclave (enclave-based justice)

Moshe Gorali ; "Legality in the eye of the beholder", haaretz, article non daté

14. Adriana Kemp; " Naissance d'une «minorité piégée » : la gestion de la population arabe dans les débuts de l'Etat d'Israël »; Critiques Internationales, 2002, 2 (15), p.105-124

15. Rapport de l'Association Arabe pour les Droits de l'Homme (Arab Association for Human Rights), juin 2002 
16. Avigdor Lieberman, le leader charismatique d'Yisrael beitanu, un parti ancré à droite et bien implanté dans la communauté russophone, a depuis mai 2004, associé son nom à ce programme de transfert territorial.

17. Sur ce point, les études quantitatives effectuées par Sammy Smooha ne laissent guère de place au doute.

Sammy Smooha; Index of Arab-Jewish relations in Israel; the Jewish-Arabe Center, Haifa University, 2004

18. Le tracé du mur de séparation ne suivant pas la Ligne Verte (la frontière - border de 1967), il laisse un certain nombre de villages palestiniens entre le Mur et la Ligne Verte.

\section{RÉSUMÉS}

Construit sur l'opposition entre les concepts anglo-saxons de border et de frontier, cet article tente de montrer en quoi la construction par Israël du « mur de séparation » n'a pas fait émerger une frontière de type border. Des deux côtés de la Ligne Verte, les pratiques territoriales israéliennes demeurent inféodées à une logique de frontier. Dans cette logique, c'est l'implantation d'un espace par une population ethniquement identifiée au groupe pionnier qui constitue cet espace en territoire étatique. L'existence d'une frontier ouverte contribue ainsi à inverser le rapport classique entre population et territorialité. Le demos, le peuple, n'est pas défini territorialement comme l'ensemble des individus résidant sur le territoire de l'Etat; il est défini au préalable, sur des considérations ethniques, et c'est sa dispersion spatiale qui construit la territorialité étatique.

Built on the opposition between the concepts of border and frontier, this article is aimed at showing that the construction of the separation fence does not signify the emergence of a border. On both sides of the Green Line, Israeli territorial practises are still expressing a frontier logic. In this logic, it's the settlement of a space by a population ethnically identified with the pioneer group that makes this space a State territory. The existence of an open frontier thus contributes to invert the classical link between population and territoriality. The demos, the people, is not defined territorially as the individuals residing in the State territory; it is rather defined ethnically and it's its spatial dispersion that builds the State territory.

\section{INDEX}

Mots-clés : Israël, Palestiniens, pionniers, frontières, citoyenneté

Keywords : Palestinians, pioneers, frontiers, citizenship

\section{AUTEUR}

\section{PIERRE RENNO}

Pierre Renno est doctorant en Sciences Politiques à l'Université Paris I et actuellement titulaire d'une bourse d'aide à la recherche au Centre de Recherche Français de 
Jérusalem. Il a récemment publié : « L'introuvable déségrégation ethnique des villages communautaires galiléens »; Bulletin du Centre de Recherche Français de Jérusalem, 2006, $\mathrm{n}^{\circ}$ 17 ; Disponible en ligne sur revue.org : http://bcrfj.revues.org/document246.html L'entrée « les deux Intifada » de l'encyclopédie de la culture politique contemporaine, sous la direction d'Alain Renaut ; sous presse - publication prévue en avril 2008 aux éditions Hermann 